\title{
CONTROL DESIGN FOR ENERGY SAVING
}

\author{
Xavier BRUN, Daniel THOMASSET, Serge SCAVARDA \\ Laboratoire d'Automatique Industrielle \\ http://csiges 7.insa-lyon.fr/lai/lai gb.html \\ INSA Lyon, Bâtiment Saint Exupéry \\ 25 Avenue Jean Capelle, 69621 Villeurbanne Cedex, France \\ Tel: (33) $472438881 \quad$ Fax: (33) 472438535 \\ email: xavier.brun@lai.insa-lyon.fr
}

\begin{abstract}
This paper presents the interest of including control strategy into electropneumatic design. For this certain experimental results obtained in an electropneumatic process have been presented. Using two servo-distributors leads to a system with one degree of freedom. This opportunity is exploited for in two objectives. The first concerns positioning control and the second attempts to optimise energy efficiency. With this aim an energy optimisation algorithm has been presented. The chosen control algorithm issues from the flatness concept.
\end{abstract}

\section{KEY WORDS}

nonlinear control, flatness, energy saving, electropneumatic

\section{NOMENCLATURE}

acceleration $\left(\mathrm{m} / \mathrm{s}^{2}\right)$

viscous coefficient $(\mathrm{N} / \mathrm{m} / \mathrm{s})$

heat transfer exchange coefficient $(\mathrm{J} / \mathrm{kg} / \mathrm{K})$

force $(\mathrm{N})$

$H$ enthalpy mass flow rate delivered inside chambers $(\mathrm{J} / \mathrm{s})$

jerk $\left(\mathrm{m} / \mathrm{s}^{3}\right)$

polytropic constant

moving load $(\mathrm{kg})$

pressure $(\mathrm{Pa})$

$\mathrm{q}_{\mathrm{m}}$ mass flow rate $(\mathrm{kg} / \mathrm{s})$

perfect gas constant related to unit mass $(\mathrm{J} / \mathrm{kg} / \mathrm{K})$

area of the piston cylinder $\left(\mathrm{m}^{2}\right)$

time (s)

temperature $(\mathrm{K})$

servo-distributor input voltage $(\mathrm{V})$

velocity $(\mathrm{m} / \mathrm{s})$

volume $\left(\mathrm{m}^{3}\right)$

$W_{P N E U}^{\text {in }}$ pneumatic energy which enters in actuator $(\mathrm{J} / \mathrm{s})$

$\underline{\mathrm{x}} \quad$ state vector

y position (m)

$\varphi(),. \psi($.) and $\Gamma($.$) are polynomial functions$

\begin{tabular}{ll}
\multicolumn{2}{l}{ Subscripts and superscripts } \\
cyl & cylinder \\
d & desired \\
ext & external \\
f & dry friction \\
$\mathrm{N}$ & chamber N \\
$\mathrm{P}$ & chamber P \\
S & supply pressure \\
d & desired \\
in & input (positive value) \\
out & output (negative value)
\end{tabular}

\section{INTRODUCTION}

At the present time, one five-way servo-valve or servodistributor control many actuators for position or velocity tracking. The tendency of building longer rodless cylinders is one of the principal reasons for the development of three-way electropneumatic modulators. In fact the integration of this component in each cylinder extremity reduces the congestion and the length of the pneumatic pipes. This technology has never been developed for control strategies. Indeed, the constructors usually use these two three-way modulators with the same air power supply and the same input controls but with opposite signs, which is equivalent to using one five-way servo-distributor. This paper presents the interest of including the control strategy into electropneumatic design. Energy economy has been developed with electrical actuators (asynchronous motors) [1], however for solutions envisaged in fluid power the proposition is to modify the structure of the system [2]. Here the proposed solution modifies both the system design and the control architecture.

It would seem interesting to design an inline actuator with two servo-distributors controlled by two different control laws. Taking advantage of the degree from freedom issued of this new design, it is possible to control another output other than the position control. In our approach the second output is the pressure in one chamber, from which depends the energy delivered by the system. Thus it would seem possible to respect two different objectives, with two dissociated controls. The 
aim is also to decrease the necessary energy without reducing the performance in terms of position tracking.

The results presented in this paper come from nonlinear control strategy. Experimental results originating from monovariable and multivariable control laws are compared. The first criterion concerns the tracking performance. The new strategy does not decrease tracking errors, nor the static errors, nor the standard deviation in term of position and velocity tracking. The second criterion concerns the quantity of necessary energy for these strategies. Today, this point is an important line of research. Due to poor efficient of electropneumatic actuators, a lot of progress in terms of energy consumption seems to be possible. Now this method has been developed in the electropneumatic field today but is similar for electrohydraulic applications where the financial gain will be greater.

\section{ELECTROPNEUMATIC MODELING PART}

The system under consideration (Figure 1) is an in-line electropneumatic servodrive controlled by two threeway servo-distributors. The stroke length is half a meter and the total moving load is $17 \mathrm{~kg}$.

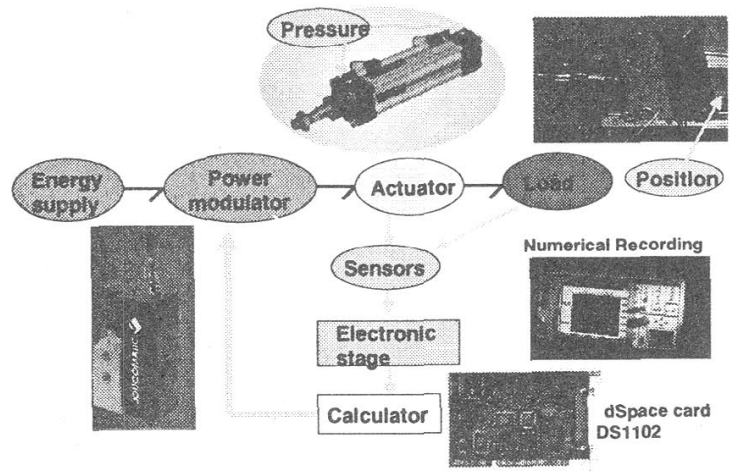

Figure 1 Electropneumatic system

The electropneumatic system model can be obtained using classical assumptions [3]: by considering the pressure behaviour in a chamber with variable volume and the mechanical equation which includes pressure force, viscous friction (b.v), dry friction $\left(F_{f}\right)$ and an external force $\left(F_{\text {ext }}\right)$ due to atmospheric pressure. Using the theory of multi-scale systems, the dynamics of the servo-valves can be neglected [4]. So the model can be reduced to a static one described by two relationships $q_{m}{ }_{P}\left(u_{P}, p_{P}\right)$ and $q_{m}{ }_{N}\left(u_{N}, p_{N}\right)$ between the mass flow rates $q_{m} P$ and $q_{m} N$, the input voltages $u_{P}$ and $u_{N}$, and the output pressures $p_{P}$ and $p_{N}$. The state model is given by Eq. (1):

$$
\left\{\begin{array}{l}
\frac{d p_{P}}{d t}=\frac{k \cdot r \cdot T_{S}}{V_{P}(y)}\left[q_{m}\left(u_{P}, p_{P}\right)-\frac{S_{P}}{r T_{S}} \cdot p_{P} \cdot v\right] \\
\frac{d p_{N}}{d t}=\frac{k \cdot r \cdot T_{S}}{V_{N}(y)}\left[q_{m_{N}}\left(u_{N}, p_{N}\right)+\frac{S_{N}}{r T_{S}} \cdot p_{N} \cdot v\right] \\
\frac{d v}{d t}=\frac{1}{M}\left[S_{P} \cdot p_{P}-S_{N} \cdot p_{N}-b \cdot v-F_{f}-F_{e x t}\right] \\
\frac{d y}{d t}=v
\end{array}\right.
$$

In the case of a nonlinear control synthesis, the theory that respects Isidori's work [5] requires that the model must be a linear function of the control input in order to obtain an explicit form of the control law. Therefore the mass flow rate characterisation obtained from experimental measurements was approximated by a polynomial function [6] affine in control (Eq. (2)).

$$
\left\{\begin{array}{l}
q_{m}\left(u_{P}, p_{P}\right)=\varphi\left(p_{P}\right)+\psi\left(p_{P}, \operatorname{sgn}\left(\Gamma\left(u_{P}\right)\right)\right) \cdot \Gamma\left(u_{P}\right) \\
q_{m}\left(u_{N}, p_{N}\right)=\varphi\left(p_{N}\right)+\psi\left(p_{N}, \operatorname{sgn}\left(\Gamma\left(u_{N}\right)\right)\right) \Gamma\left(u_{N}\right)
\end{array}\right.
$$

$\varphi($.) and $\psi($.) are polynomial functions and $\Gamma($.) is an odd function.

With two inputs $\Gamma\left(u_{P}\right)$ and $\Gamma\left(u_{N}\right)$, the nonlinear model of the system has the following form (Eq. (3)):

$$
\underline{x}=f(\underline{x})+G(\underline{x}) \underline{U}
$$

where $f(x), G(x)$ and $\underline{U}$ are given by relations (4).

$$
\begin{gathered}
\underline{x}=\left[\begin{array}{c}
p_{P} \\
p_{N} \\
v \\
y
\end{array}\right] \quad f(\underline{x})=\left(\begin{array}{c}
\frac{k r T_{s}}{V_{P}(y)}\left[\varphi\left(p_{P}\right)-\frac{S_{P}}{r T_{s}} p_{P} v\right] \\
\frac{k r T_{s}}{V_{N}(y)}\left[\varphi\left(p_{N}\right)+\frac{S_{N}}{r T_{s}} p_{N} v\right] \\
\frac{1}{M}\left[S_{P} p_{P}-S_{N} p_{N}-b v-F_{f}-F_{\text {ext }}\right] \\
v
\end{array}\right) \\
G(x)=\left(\begin{array}{cc}
\frac{k . r . T_{S}}{V_{P}(y)} \cdot \psi\left(p_{P}, \operatorname{sgn}\left(U_{P}\right)\right) & 0 \\
0 & \left.\frac{k \cdot r \cdot T_{S}}{V_{N}(y)} \cdot \psi\left(p_{N} \cdot s g n\left(U_{N}\right)\right)\right) \\
0 & 0 \\
0 & 0
\end{array}\right) \underline{U}=\left(\begin{array}{l}
U_{P} \\
U_{N}
\end{array}\right)=\left(\begin{array}{l}
\Gamma\left(u_{P}\right) \\
\Gamma\left(u_{N}\right)
\end{array}\right)
\end{gathered}
$$

\section{OPTIMIZATION ALGORITHM}

The control strategy relates to tracking the desired position with the minimum energy consumption. For this, a primary study in simulation has been carried out [7] to evaluate the possibility of improving the efficiency with an appropriate supplementary desired trajectory. To reduce the energy consumed, it is possible to decrease the mass flow rate delivered by each servovalve. So the choice is to elaborate a desired pressure trajectory in chamber $P$. This solution is easy to implement and does not need an additional sensor. In order to do this, an optimisation algorithm has been developed.

The pneumatic energy delivered by the compressor that enters in the actuator through each servodistributor is 
given by Eq. (5). This energy is denoted $W_{P N E U}^{\text {in }} ; H_{P_{i n}}$ and $H_{N_{i n}}$ are the enthalpy mass flow rates delivered respectively inside chambers $\mathrm{P}$ and $\mathrm{N}$ of the actuator. $\mathrm{c}_{\mathrm{p}}$ is the constant pressure specific heat and $T_{S}$ is the temperature supply (assumed constant).

$$
W_{P N E U}^{\text {in }}=\int_{0}^{T_{\text {ock }}}\left[H_{P_{i n}}(t)+H_{N_{i n}}(t)\right] d t \text { with }\left\{\begin{array}{l}
H_{p_{m}}(t)=q_{m}\left(u_{P}(t) p_{P}(t)\right) c_{p} T_{s} \\
H_{N_{i n}}(t)=q_{m}\left(u_{N}(t), p_{N}(t)\right) \mathrm{c}_{p} T_{S}
\end{array}\right.
$$

Another important argument that led us to choose the pressure as the second output, is its flatness property [8]. Indeed, position and pressure are flat outputs (see following part), and so the complexity of implementing the nonlinear control law will be reduced.

The flatness property means that the control law can be expressed from the desired trajectories and their successive derivations. So with this result and using Eq. (2) and (5) the enthalpy mass flow rate can be expressed with only the desired position, velocity, acceleration, jerk (imposed by the industrial specifications) pressure in one chamber and the derivation of this pressure. This is shown in Eq. (6).

$$
H_{P_{\text {in }}}(t)+H_{N_{\text {in }}}(t)=\Lambda\left(y^{d}, v^{d}, a^{d}, j^{d}, p_{P}^{d}, d p_{P}^{d} / d t\right)
$$

This function $\Lambda$ has been optimised with the constraint given by relation (7) and with the desired trajectories in position and successive derivatives given by figure 2 .

$\left\{\begin{array}{l}10^{5} \mathrm{~Pa} \leq p_{P} \leq 7.10^{5} \mathrm{~Pa} \\ 10^{5} \mathrm{~Pa} \leq p_{N} \leq 7.10^{5} \mathrm{~Pa}\end{array} \quad\right.$ and $\quad\left\{\begin{array}{l}-10 \text { volt } \leq u_{P} \leq 10 \text { volt } \\ -10 \text { volt } \leq u_{N} \leq 10 \text { volt }\end{array}\right.$

The results obtained after optimisation with constraints are presented in figure 2 .

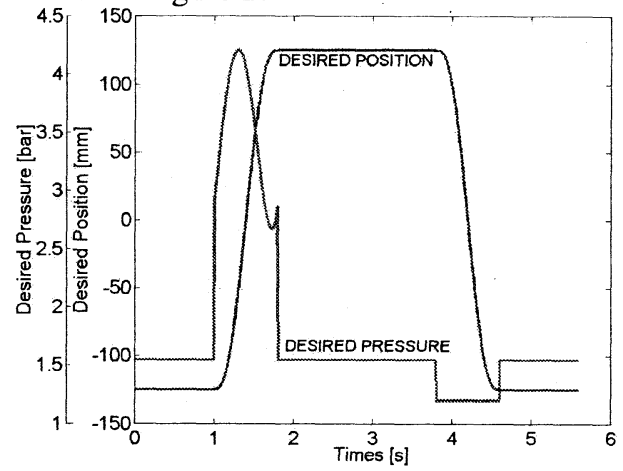

Figure 2 Desired optimised pressure in chamber $P$ for a desired position given by the specifications

\section{NONLINEAR CONTROL LAW SYNTHESIS}

So the two chosen outputs are the pressure in chamber $P$ and the position (Eq. (8)):

$$
h(x)=\left(\begin{array}{l}
h_{1}(x) \\
h_{2}(x)
\end{array}\right)=\left(\begin{array}{c}
p_{P} \\
y
\end{array}\right)
$$

The characteristic numbers associated to the pressure $p_{P}$ and the position $y$ are respectively one and three. Thus, the sum is equal to the dimension of the system. This is sufficient to affirm that the system is differentially flat [8]. Due to the flatness definition, the inputs can be written as functions of outputs and a finite number (equal to the characteristic number) of their time derivatives. A direct way to obtain the nonlinear linearizing control laws which decouple the system consists of using the expressions $d p_{P}^{d} / d t$ and $j^{d}$ and inverting them. The result is given by equation (9). With the two previous control inputs $u_{P}$ and $u_{N}$, the nonlinear electropneumatic model is transformed into two linear models, one simple integrator and one triple integrator. Classic feedback laws (see equation (10)) can stabilise each of them. In this case the global stability is obvious. This is one of the main advantages of flatness.

$$
\begin{aligned}
& u_{P}=\Gamma^{-1}\left[\frac{\frac{S_{P}}{r T_{S}} p_{P}^{d} \nu^{d}-\varphi\left(p_{P}^{d}\right)}{\psi\left(p_{P}^{d}, \operatorname{sgn}\left(U_{P}\right)\right)}+\frac{\frac{V_{P}\left(y^{d}\right)}{k r T_{S}}}{\psi\left(p_{P}^{d}, \operatorname{sgn}\left(U_{P}\right)\right)^{w_{1}}}\right] \\
& \left\{u_{N}=\Gamma^{-1}\left[-\frac{\frac{S_{N}}{r T_{S}} p_{N}^{d} \nu^{d}+\varphi\left(p_{N}^{d}\right)+\frac{V_{N}\left(y^{d}\right)}{k r T} \frac{b}{S_{N}} a^{d}}{\psi\left(p_{N}^{d}, \operatorname{sgn}\left(U_{N}\right)\right)}+\right.\right. \\
& \left.\frac{\frac{V_{N}\left(y^{d}\right)}{k r T s}}{\psi\left(p_{N}^{d}, \operatorname{sgn}\left(U_{N}\right)\right)}\left(-\frac{S_{P}}{S_{N}} w_{1}+\frac{M}{S_{N}} w_{2}\right)\right] \\
& \text { With } p_{N}^{d}=\frac{1}{S_{N}}\left(S_{P} p_{P}^{d}-b v^{d}-F_{f}-F_{e x t}-M a^{d}\right) \\
& \left\{\begin{array}{l}
w_{1}=\frac{d p_{P}^{d}}{d t}-K_{p_{P}}\left(p_{P}-p_{P}^{d}\right) \\
w_{2}=j^{d}-K_{a}\left(a-a^{d}\right)-K_{v}\left(v-v^{d}\right)-K_{y}\left(y-y^{d}\right)
\end{array}\right.
\end{aligned}
$$

\section{EXPERIMENTAL RESULTS}

The nonlinear multivariable control law has been implemented with a dSpace DS1102 acquisition card. The following experimental results are presented in terms of repeatability. The curves obtained are the arithmetical mean value for 30 cycles. The tracking position quality (Figure 3) is similar to the results obtained in the case of the monovariable nonlinear control law [9].

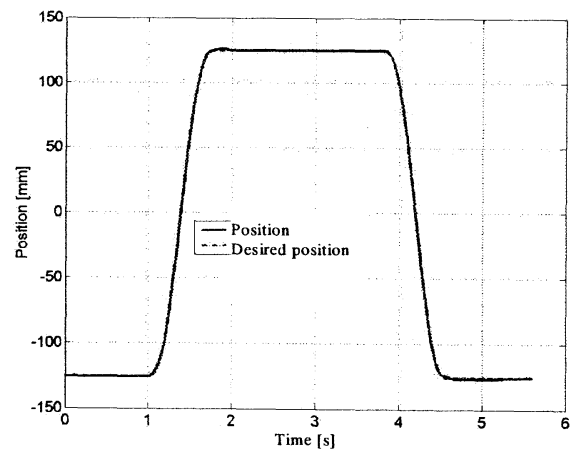

Figure 3 Tracking position results

The mean position error (Figure 4) obtained for 30 cycles is never more than $0.3 \mathrm{~mm}$ in the static stage. 
This error, during the dynamic stage, depends if the rod cylinder exits from the cylinder or enters. In the first case the maximum error value is $5.4 \mathrm{~mm}$ and becomes $3.4 \mathrm{~mm}$ in the other case. The standard deviation is 0.48 $\mathrm{mm}$ in the static stage and $0.56 \mathrm{~mm}$ in the dynamic stage, so good repeatability is assured all along the desired trajectory.

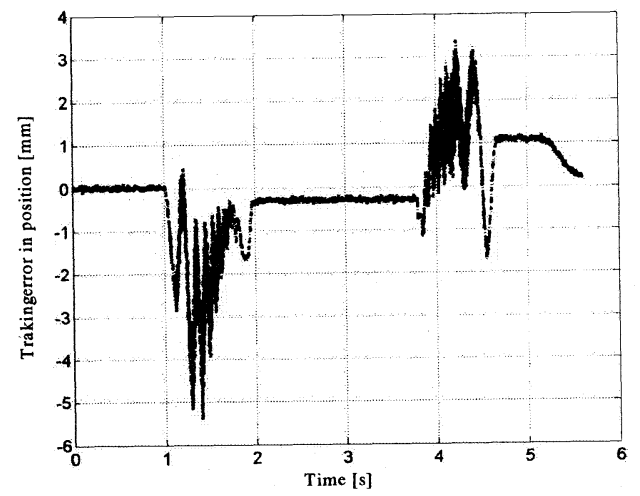

Figure 4 Tracking position error

The experimental results concerning tracking pressure trajectory are shown in figure 5. Using a dry friction model with an optimization algorithm reduces the problem due to dry friction while starting and stopping during the dynamic stage. The tracking pressure errors outside these stages are not superior to 0.1 bar for rod exit and 0.3 bar for rod entry.

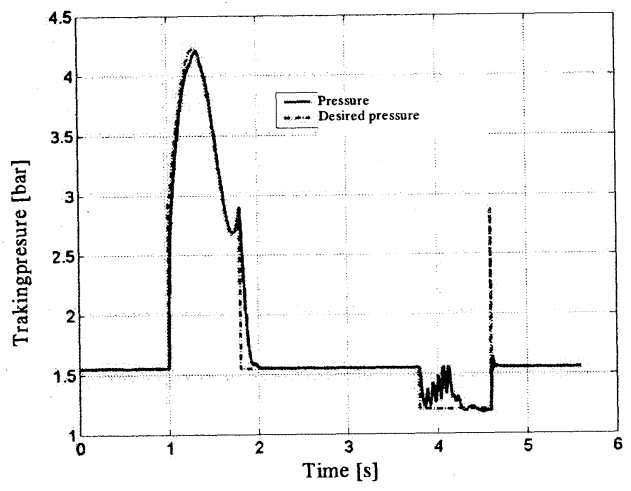

Figure 5 Pressure tracking result

In order to obtain some information about the energy consumption, the mass flow rate delivered by the two servo-distributors must be reconstructed. For this, by considering Eq. (2) and the experimental pressure and control results, it is possible to deduce the mass flow consumption during the whole movement. Considering that the equilibrium point at the start is the same that at the end, the total energy delivered by the compressor is equal to the integral of this reconstructed mass flow rate multiplied by a constant coefficient (see Eq. (5)). The numerical results obtained show an improvement of $29 \%$ in terms of the energy delivered inside the cylinder with multivariable nonlinear control.

\section{CONCLUSION}

The marked asymmetry caused by the rod is the first explication of the asymmetrical experimental results which depends on the direction of movement. That is why it is primordial to take stock of the energy consumption during a complete cycle. These first experimental results have, as a consequence an energy improvement near to $30 \%$. And these results have been obtained without degrading the tracking position or the velocity performance. Another very useful improvement concerns the global stability property of the nonlinear multivariable control law elaborated here. This is not the case for a monovariable control law [10].

The nonlinear multivariable control law seems to be a useful possibility for improving the energy efficiency of electropneumatic actuators. In order to achieve this, the control specialist has to choose to act on the structure before working on an elaborate control strategy. This study proves the importance of including the control specifications during the designing phase. The next step will consist of comparing the cost of the supplementary servodistributor with the financial gain due to the energy saving process.

\section{REFERENCES}

1. Canudas de Wit, C., Seleme, S.I. Robust Torque Regulation for Induction Motors: The Minimum energy approach. Automatica, 33-1, pp 63-79, 1997.

2. Bachmann, J.R., Surgenor, B.W. On design and performance of a closed circuit pneumatic positioning system. The fifth Scandinavian International Conf. on Fluid Power, Linköping, Sweden, Vol.1, pp 309-322, May 28-30, 1997.

3. Shearer, J.L. (1956) Study of pneumatic processes in the continuous control of motion with compressed air. Parts I and II. Trans. Am. Soc. Mech. Eng., 78, 233-249.

4. Bouhal, A. (1994) Contribution à la commande linéaire et non linéaire adaptative des systèmes électropneumatiques. Ph.D. Thesis: INSA de Lyon.

5. Isidori, A., (1989) Nonlinear control systems. New York : Springer Verlag, $2^{\text {nd }}$ edition, $479 \mathrm{p}$.

6. Belgharbi, M., Thomasset, D., Scavarda, S., \& Sesmat, S. (1999) Analytical model of the flow stage of a pneumatic servodistributor for simulation and nonlinear control., $6^{\text {th }}$ Scandina. Int. Conf. on Fluid Power, SICFP'99 (pp. 847-860). Tampere, Finland.

7. Brun, X., Thomasset, D., Sesmat, \& S., Scavarda, S. (1999b) Limited energy consumption in positioning control of electropneumatic actuator. Bath Workshop on Power Transmission \& Motion Control (pp 199-211). Bath, England.

8. Fliess, M., Levine, J., Martin, P., \& Rouchon, P. (1995) Flatness and defect of non-linear systems : introductory, theory and applications. International Journal of Control, 61, 1327-1361.

9. Brun X., Belgharbi M., Sesmat S., Thomasset D., Scavarda S., Control of an electropneumatic actuator, comparison between some linear and nonlinear control laws, Jour. of Syst. and Control Engineering, 1999, Vol. 213, N I5, p 387-406.

10. Richard, E., Scavarda, S., "Comparison between Linear and Nonlinear control of an eletropneumatic servodrive." Jour. of Dyn. Syst. Meas. \& Control, June 1996, Vol. 118, p 245-252. 